\title{
Mechanisms Suppressing Superheavy Element Yields in Cold Fusion Reactions
}

\author{
K. Banerjee, ${ }^{1, *}$ D. J. Hinde,${ }^{1, \dagger}$ M. Dasgupta, ${ }^{1}$ E. C. Simpson, ${ }^{1}$ D. Y. Jeung, ${ }^{1}$ C. Simenel, ${ }^{1}$ B. M. A. Swinton-Bland, ${ }^{1}$ \\ E. Williams, ${ }^{1}$ I. P. Carter, ${ }^{1, \$}$ K. J. Cook, ${ }^{1}$ H. M. David, ${ }^{2}$ Ch. E. Düllmann, ${ }^{2,3,4}{ }^{\text {J. Khuyagbaatar, }}{ }^{2,3}$ B. Kindler, ${ }^{2}$ B. Lommel, ${ }^{2}$ \\ E. Prasad, ${ }^{1, \S}$ C. Sengupta, ${ }^{1}$ J. F. Smith, ${ }^{1, \|}$ K. Vo-Phuoc, ${ }^{1}$ J. Walshe, ${ }^{1}$ and A. Yakushev ${ }^{2,3}$ \\ ${ }^{1}$ Department of Nuclear Physics, Research School of Physics and Engineering, The Australian National University, \\ Canberra ACT 2601, Australia \\ ${ }^{2}$ GSI Helmholtzzentrum für Schwerionenforschung, 64291 Darmstadt, Germany \\ ${ }^{3}$ Helmholtz Institute Mainz, 55099 Mainz, Germany \\ ${ }^{4}$ Institut für Kernchemie, Johannes Gutenberg-Universität Mainz, 55099 Mainz, Germany
}

(Received 13 July 2018; revised manuscript received 17 October 2018; published 14 June 2019)

\begin{abstract}
Superheavy elements are formed in fusion reactions which are hindered by fast nonequilibrium processes. To quantify these, mass-angle distributions and cross sections have been measured, at beam energies from below-barrier to $25 \%$ above, for the reactions of ${ }^{48} \mathrm{Ca},{ }^{50} \mathrm{Ti}$, and ${ }^{54} \mathrm{Cr}$ with ${ }^{208} \mathrm{~Pb}$. Moving from ${ }^{48} \mathrm{Ca}$ to ${ }^{54} \mathrm{Cr}$ leads to a drastic fall in the symmetric fission yield, which is reflected in the measured massangle distribution by the presence of competing fast nonequilibrium deep inelastic and quasifission processes. These are responsible for reduction of the compound nucleus formation probablity $P_{C N}$ (as measured by the symmetric-peaked fission cross section), by a factor of 2.5 for ${ }^{50} \mathrm{Ti}$ and 15 for ${ }^{54} \mathrm{Cr}$ in comparison to ${ }^{48} \mathrm{Ca}$. The energy dependence of $P_{\mathrm{CN}}$ indicates that cold fusion reactions (involving ${ }^{208} \mathrm{~Pb}$ ) are not driven by a diffusion process.
\end{abstract}

DOI: 10.1103/PhysRevLett.122.232503

The development of the shell model of nuclear structure resulted in the prediction [1-3] in the 1960s of an "island" of enhanced stability for nuclei up to $30 \%$ more massive than the heaviest naturally occurring elements. The predicted existence of these superheavy elements (SHE) results from closed shells of protons and/or neutrons (socalled magic numbers) that provide increased nuclear stability - analogous to that of noble gases in chemistry. Different models predict the island may be centred at proton numbers $Z=114,120$, or 126 [1-4]. The synthesis of all SHE up to $Z=118$, and their long half-lives (up to seconds), indicate the existence of the island [5], but its center and extent are not yet known. Defining the properties of SHE, and the location of the boundary to the existence of the chemical elements, are major goals in physics and chemistry.

SHE are created in the laboratory through the fusion of two lighter nuclei. This has required impressive experimental advances due to the extremely small production cross sections in the order of $10^{-36} \mathrm{~cm}^{2}$. Fundamentally these result from the Coulomb repulsion between the large number of protons that must be packed together in the SHE nucleus. Even with sufficient kinetic energy to bring the surfaces of the colliding nuclei into contact, fast nonequilibrium deep-inelastic (DIC) [6,7] and quasifission (QF) [8] processes can cause the system to reseparate so quickly $\left(<10^{-20} \mathrm{~s}\right)$ that a compact superheavy nucleus is not formed, thus suppressing fusion. Even if the two nuclei fuse and form a compact compound nucleus $(\mathrm{CN})$, the heavy element is unlikely to survive, since the $\mathrm{CN}$ usually splits (fissions) in $<10^{-16} \mathrm{~s}$ into two similar-sized fragments (fusion fission $[\mathrm{FF}]$ ).

The most successful fusion reactions have used projectile and/or target nuclei that have magic numbers of protons $(Z)$ and/or neutrons $(N)$, particularly ${ }^{48} \mathrm{Ca}(Z=20, N=28)$ and ${ }^{208} \mathrm{~Pb}(Z=82, N=126)$. Their use, rather than nearby nuclei, results in lower excitation energy of the fused system, reducing the probability of fission. For this reason, fusion of heavy nuclei with ${ }^{208} \mathrm{~Pb}$ is known as cold fusion. Recent experiments [9-12] have indicated that collisions of nuclei having several magic numbers not only decreases the probability of fission after fusion, but also increases the probability of fusion itself.

The heaviest element Oganesson $(Z=118)$ was formed by fusing accelerated ${ }^{48} \mathrm{Ca}$ nuclei with radioactive ${ }^{249} \mathrm{Cf}$ target nuclei [13]. To form even heavier elements, projectiles with more protons than ${ }^{48} \mathrm{Ca}$ must be used because of the near impossibility of creating enough target material of elements heavier than $\mathrm{Cf}$ [14]. Their use results in lower SHE yield, as demonstrated by the unsuccessful attempts to synthesize element $Z=120$ [15] with ${ }^{50} \mathrm{Ti},{ }^{54} \mathrm{Cr}[16],{ }^{58} \mathrm{Fe}$ [17], and ${ }^{64} \mathrm{Ni}[18]$ beams. It is vital to understand the reaction dynamics in order to choose the best reactions to produce new SHE.

Because of the different timescales of the three stages of SHE synthesis, namely contact, fusion, and fusion fission, 
the SHE cross section $\sigma_{\mathrm{SHE}}$ is written [19] as the product of three terms, summed over all angular momenta $(J \hbar)$ :

$$
\sigma_{\mathrm{SHE}}(E)=\sum_{J=0}^{\infty} \sigma_{c}(E, J) P_{\mathrm{CN}}(E, J) W_{s u r}\left(E_{x}, J\right)
$$

Here $\sigma_{c}$ is the capture (contact) cross section at a center of mass energy $E$. $P_{\mathrm{CN}}$ is the probability of surviving fast reseparation, resulting in fusion-the formation of a compact compound nucleus. $W_{\text {sur }}$ is the probability that after fusion the compound nucleus survives fission decay. $W_{\text {sur }}$ is expected to fall rapidly with increasing $J$ and excitation energy $E_{x}$ [20]. Among these factors $P_{\mathrm{CN}}$ is the least understood theoretically. Different models assume different variables determine $P_{\mathrm{CN}}$, such as mass asymmetry, or elongation, or both, in time-independent or dynamical approaches, including diffusion [19,21,22]. Their predictions can differ by orders of magnitude $[23,24]$. This makes it challenging to optimize experiments to produce new elements and isotopes.

$P_{\mathrm{CN}}$ can be investigated experimentally (for limited cases) by measuring $\sigma_{\mathrm{SHE}}$ for different reactions forming the same compound nucleus [10,25-28]. Although eliminating sensitivity to $W_{\text {sur }}$, the measurements are often difficult due to low cross sections, and do not give direct information on the processes that suppress fusion and thus control the value of $P_{\mathrm{CN}}$.

This Letter maps out the evolution of the yields and characteristics of the processes that compete with fusion, and investigates $P_{\mathrm{CN}}$ values in cold fusion reaction with ${ }^{208} \mathrm{~Pb}$, for projectiles $\mathrm{Ca}\left(Z_{P}=20\right)$, $\mathrm{Ti}\left(Z_{P}=22\right)$, and $\mathrm{Cr}\left(Z_{P}=24\right)$.

Measurements were carried out at the Heavy Ion Accelerator Facility of the Australian National University. Isotopically enriched targets of ${ }^{208} \mathrm{PbS}, \sim 210 \mu \mathrm{g} / \mathrm{cm}^{2}$ in thickness on $30 \mu \mathrm{g} / \mathrm{cm}^{2} \mathrm{C}$ backings, were bombarded by pulsed beams (full width at half maximum from 0.5 to $1.5 \mathrm{~ns})$. Beam energies ranged from below the capture barriers $V_{B}$ to $25 \%$ above. Binary scattering and fission products were detected in coincidence using three positionsensitive multiwire proportional counters (MWPCs) $[29,30]$. These covered scattering angle $(\theta)$ ranges $10^{\circ} \leq$ $\theta \leq 80^{\circ}$ on one side of the beam axis, and $53^{\circ} \leq \theta \leq 169^{\circ}$ on the other side. Further details of the experiment are given in the Supplemental Material [31].

For binary reaction outcomes, the mass-ratio $M_{R}$ (defined as the mass of one fragment divided by the summed masses of both fragments) and center-of-mass angle $\theta_{\text {c.m. }}$. were determined from the time-of-flight and position information from the MWPCs. Plotting $M_{R}$ and $\theta_{\text {c.m. }}$ for each event generated a mass-angle distribution (MAD) for each beam energy [30,34]. Figure 1 shows examples of MADs for the three reactions at $\sim 0.99 V_{B}$, $1.07 V_{B}$, and $1.11 V_{B}$. The highest energy measurement for ${ }^{54} \mathrm{Cr}$ used only two MWPC detectors [11].
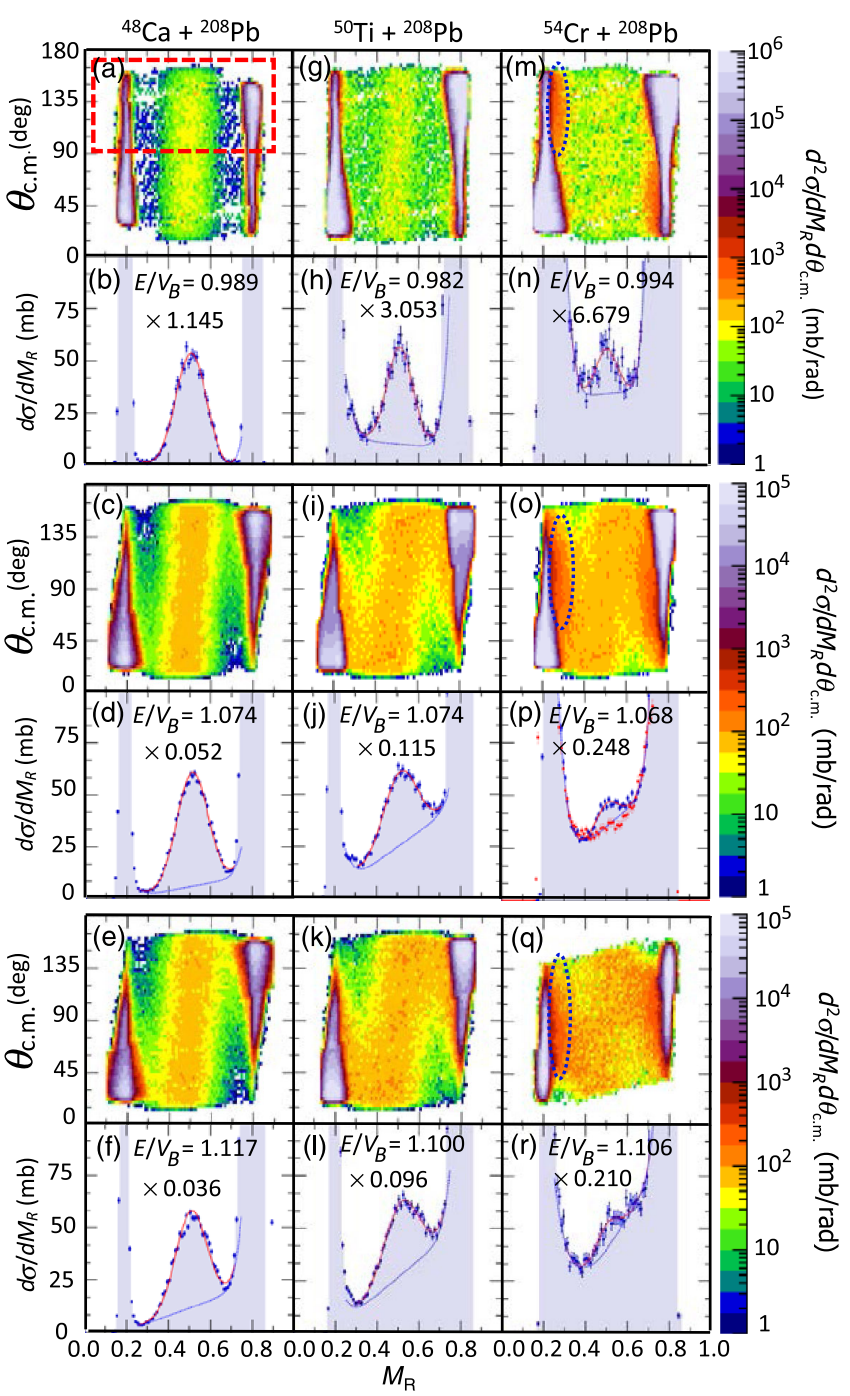

FIG. 1. Mass angle distributions (MADs) and (below) projected mass ratio spectra within the red dashed gate in (a), for ${ }^{48} \mathrm{Ca},{ }^{50} \mathrm{Ti}$, and ${ }^{54} \mathrm{Cr}$ reactions with ${ }^{208} \mathrm{~Pb}$ at the values of $E / V_{B}$ indicated. The experimental cross sections have been multiplied by the factor given for each measurement in the projection panel for clarity in presenting the results. Blue curves are quasifission background (see text). ${ }^{54} \mathrm{Cr}+{ }^{204} \mathrm{~Pb}$ data at $E / V_{B} 1.08$ are shown by red symbols in (p).

The MADs show the double differential cross section $d^{2} \sigma / d M_{R} d \theta_{\text {c.m. }}$ normalized using deep subbarrier Rutherford scattering measured in the MWPCs [31]. Further details are given in the Supplemental Material [31]. The MADs show three major groups of events. The first two are intense peaks at $M_{R} \simeq 0.2$ and 0.8 . These peak at forward and backward angles, corresponding to projectilelike and targetlike nuclei, respectively. Between these groups are fissionlike events where the projectile and target nuclei have stuck together, the system has rotated through some angle, then come apart with a mass ratio closer to 0.5 . The observed angular asymmetries depend on the beam nuclide and energy, and these provide key insights into the reaction dynamics, as discussed below. 
If $d^{2} \sigma / d M_{R} d \theta_{\text {c.m. }}$ at a given $\theta_{\text {c.m. }}$ is asymmetric about $M_{R}=0.5$, this indicates a process where reseparation occurs typically faster than one rotation. If the yield is symmetric, no "memory" of the mass and direction of the reactants remains, implying a much longer timescale. Typically this corresponds to fusion fission [35,36]. These characteristics are highlighted by projections of the MADs over the limited angular range $90^{\circ} \leq \theta_{\text {c.m. }} \leq$ $170^{\circ}$ [indicated by the red dashed rectangle in Fig. 1(a)], giving the $M_{R}$ spectra below each MAD. The degree of asymmetry about $M_{R}=0.5$ is related to the fraction of short timescale events.

For the ${ }^{48} \mathrm{Ca}$ reaction at $0.99 V_{B}$ [Fig. 1(a)], the events centred at $M_{R}=0.5$, independent of angle, are consistent with fusion fission. At the higher ${ }^{48} \mathrm{Ca}$ beam energies [Figs. 1(c)-1(f)], a mass-asymmetric fission componentfast quasifission-having a strong correlation of yield with angle fills the region between mass-symmetric fission and scattering events [37]. The symmetric-peaked groups in the MADs [Figs. 1(c) and 1(e)] also show a mass-angle correlation, indicating that the quasifission events extend towards symmetric mass splits. This indicates a shorter average time scale than at $V_{B}$ and that, for ${ }^{48} \mathrm{Ca}+{ }^{208} \mathrm{~Pb}$, quasifission is making an increasing contribution to the mass-symmetric region with increasing $E$. This contrasts with a previous interpretation [38], where the peak around mass-symmetry over a limited angular range was attributed completely to fusion fission.

At the lowest energies ${ }^{50} \mathrm{Ti}$ and ${ }^{54} \mathrm{Cr}$ have weaker peaks at mass-symmetry than ${ }^{48} \mathrm{Ca}$, perhaps with a slight mass-angle correlation, suggesting a greater quasifission contribution than for ${ }^{48} \mathrm{Ca}$. There is a (small) mass-symmetric peak [Figs. 1(q) and 1(r)] for ${ }^{54} \mathrm{Cr}+{ }^{208} \mathrm{~Pb}$ even at the highest energy. However a recent study of several $\mathrm{Cr}+\mathrm{Pb}$ reactions [11] found that ${ }^{54} \mathrm{Cr}+{ }^{204} \mathrm{~Pb}$, with fewer magic numbers, had no visible symmetric fission peak [as shown by the red points in Fig. 1(p)]. This suggests that the changing yield in the symmetric peak is not only due to macroscopic factors (e.g., projectile-target charge product $Z_{P} Z_{T}$ [30]) but also microscopic properties (e.g., closed shells $[11,12])$.

Qualitatively, the data show that the region between scattering and mass-symmetric fission fills with increasing $Z_{P}$ [Figs. 1(b), 1(h), and 1(n)] as well as with $E / V_{B}$ [Figs. 1(b), 1(d), and 1(f)], corresponding to an increasing fraction of quasifission.

To carry out a quantitative evaluation of $P_{\mathrm{CN}}$ within the framework of Eq. (1), determination of the expected capture cross sections $\sigma_{c}$ is required, which needs the capture barrier energies $V_{B}$ for each reaction. The energy dependence of the fissionlike yields with a long sticking time, resulting only from capture, should allow determination of $V_{B}$. To consistently select these events, an $M_{R}$ cut between the dips separating fast (leading to massasymmetric fragments) and slower (more symmetric)

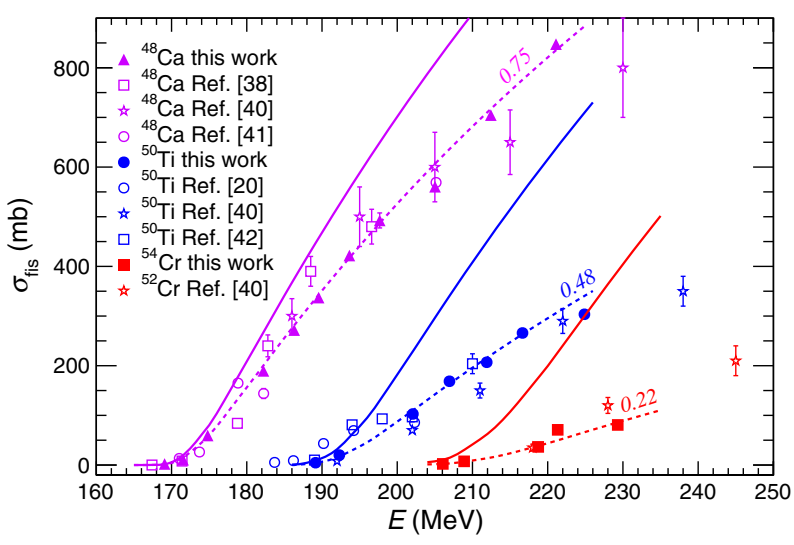

FIG. 2. Measured fissionlike cross sections (filled symbols) as a function of center-of-mass energy $E$, together with previous data (open symbols) [20,38,40-42]. Coupled channels calculations are shown by full lines. Dashed lines show these calculations scaled by the factor indicated.

reactions were applied. The mass-ratio gates used were $0.3 \leq \mathrm{M}_{R} \leq 0.7$ for ${ }^{48} \mathrm{Ca},{ }^{50} \mathrm{Ti}$, and $0.35 \leq \mathrm{M}_{R} \leq 0.65$ for ${ }^{54} \mathrm{Cr}$. Fissionlike cross sections $\sigma_{\text {fis }}$ were determined by extrapolation of angular distribution to $180^{\circ}$ using a Transition State Model fit [39].

Figure 2 shows the measured $\sigma_{\text {fis }}$, which agree with earlier measurements $[20,38,40-42]$. The capture barrier energies $V_{B}$ were determined by fitting above barrier data with a classical barrier-passing model: $\sigma_{\mathrm{fis}}=\pi R_{B}^{2}\left(1-V_{B} / E\right)$, where $V_{B}$ and the barrier radius $R_{B}$ were free parameters, resulting in $V_{B}=173.4 \pm 0.1,192.6 \pm 0.1$, and $207.3 \pm 0.3 \mathrm{MeV}$ for ${ }^{48} \mathrm{Ca},{ }^{50} \mathrm{Ti}$, and ${ }^{54} \mathrm{Cr}$ respectively.

Capture cross sections $\sigma_{c}$ were then calculated using the quantum coupled channels code CCFULL [43], including excitations of both target and projectile nuclei [31]. The calculations were constrained to reproduce the experimental capture barriers $V_{B}$, giving the $\sigma_{c}$ shown in Fig. 2 by the full lines. The measured $\sigma_{\text {fis }}$ lie below $\sigma_{c}$, the deviation increasing with $Z_{P}$. The $\sigma_{\text {fis }}$ could be well reproduced by scaling $\sigma_{c}$ by constant factors of 0.75 for ${ }^{48} \mathrm{Ca}, 0.48$ for ${ }^{50} \mathrm{Ti}$, and 0.22 for ${ }^{54} \mathrm{Cr}$ (dashed lines in Fig. 2).

The very large reductions for the ${ }^{50} \mathrm{Ti}$ and ${ }^{54} \mathrm{Cr}$ reactions might be surprising; however a systematic analysis [44] of fusion cross sections for a large number of reactions up to $Z_{P} Z_{T}=1600$ showed a reduction in fusion with increasing $Z_{P} Z_{T}$. A scaling factor of 0.75 for ${ }^{48} \mathrm{Ca}$ is consistent with these systematics. Scaling factors smaller than 0.7 would be expected for ${ }^{50} \mathrm{Ti}$ and ${ }^{54} \mathrm{Cr}\left(Z_{P} Z_{T}>1800\right)$. The reduction in capture is not due to the different Coulomb potentials, as this is taken into account in the CCFULL calculations. It was suggested in Ref [44] that deep inelastic processes might be responsible. If kinetic energy were dissipated into internal (thermal) excitation before reaching the barrier, the system could be reflected from the capture barrier, thus suppressing capture. If so, the "missing" cross section should consist of reduced energy projectilelike and 


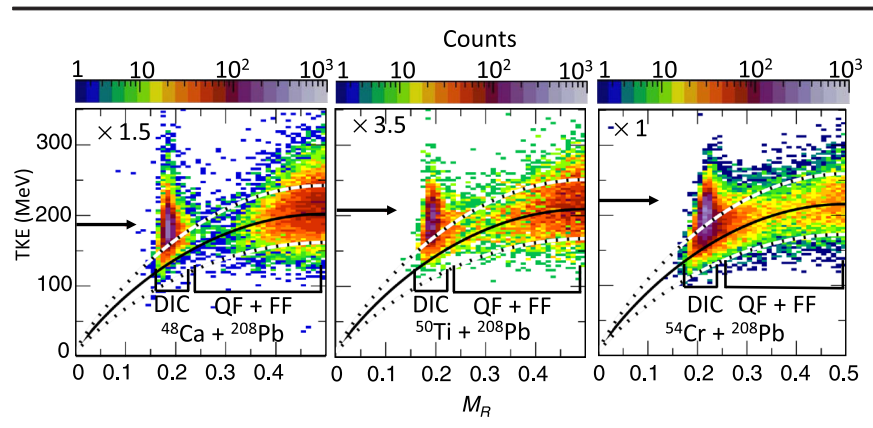

FIG. 3. Measured TKE as a function of mass ratio for ${ }^{48} \mathrm{Ca},{ }^{50} \mathrm{Ti}$, and ${ }^{54} \mathrm{Cr}$ at energies $E / V_{B} \sim 1.07$. The spectra are normalized by the indicated factor to give the same integrated counts. The angle cut $\theta_{\text {c.m. }}>135^{\circ}$ results in the elastic scattering yields (elastic energies are indicated by the arrows) being small. The curves indicate the energies for fully damped events (see text). Typical regions for DIC, QF, and FF are marked.

targetlike nuclei, and be evident in the MADs, unless masked by intense elastic scattering at forward angles. The ${ }^{54} \mathrm{Cr}$ MAD at the lowest energy [Fig. 1(m)] indeed shows a strong group of events (highlighted by the blue ellipse) having a broad spread of angles centred at $\theta_{\text {c.m. }}=135^{\circ}$, with mass flow extending to $M_{R} \sim 0.3$ from the initial $M_{R}=0.21$. The peak of this group moves forward with energy, to $\theta_{\text {c.m. }}=105^{\circ}$ and then $\theta_{\text {c.m. }}=80^{\circ}$, correlated with the expected forward movement of the grazing angle with $E / V_{B}$.

The nature of these events can be investigated through their total kinetic energies (TKE), which are also obtained with the kinematic coincidence technique $[7,8,45,46]$. Figure 3 shows TKE as a function of $M_{R}$ for the three projectiles at $E / V_{B} \sim 1.07$ and $\theta_{\text {c.m. }}>135^{\circ}$. Here the elastic scattering yield is small. Typical regions for different processes (DIC, QF, and FF) are marked. The full curves show the expectation for fully energy-damped events, based on Viola's systematics [47] for symmetric fission, extended to mass-asymmetric splits $[40,48]$. The whiteblack dashed curves indicate a range of $\pm 20 \%$.

The majority of events lie in this "fully damped" band for each reaction. However, the $M_{R}$ distributions within the band differ. For the ${ }^{48} \mathrm{Ca}$ reaction, the projectilelike events are well separated in $M_{R}$ from the more intense fissionlike events $\left(M_{R}>0.3\right)$ peaked at mass symmetry. As $Z_{P}$ increases this separation is diminished, with ${ }^{54} \mathrm{Cr}$ exhibiting a continuous distribution up to $M_{R}=0.5$, with the yield for $M_{R}>0.3$ being much smaller than for $M_{R}<0.3$. The data show that DIC and QF distributions are not separated in mass, angle, or kinetic energy but are part of a continuous spectrum of fast reaction outcomes that reduce $P_{\mathrm{CN}}$.

As the intense elastic scattering masks some of the DIC events at forward angles, extraction of total DIC cross sections is not currently possible. However it is clear that there is a strong correlation with $Z_{P}$ of both (i) the degree of suppression of $\sigma_{\text {fis }}$ compared with coupled-channels

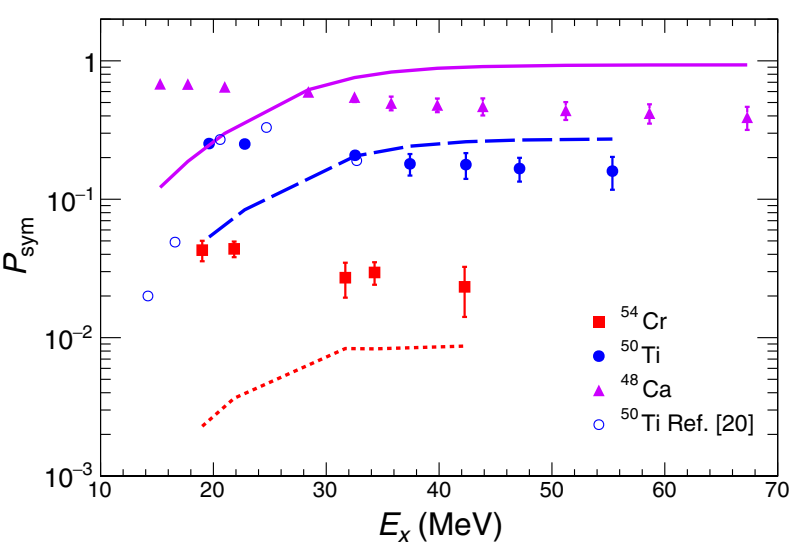

FIG. 4. Ratio of the symmetric-peaked fission yield (after subtraction of a quasifission background) to the capture cross sections, representing upper limits to $P_{\mathrm{CN}}$ (see text). Error bars include estimated background uncertainties [31]. Predictions of $P_{\mathrm{CN}}$ from Ref. [19], based on a diffusion model of fusion for reactions with ${ }^{208} \mathrm{~Pb}$, are also indicated with lines. Values of $P_{\mathrm{CN}}$ from [20] for ${ }^{50} \mathrm{Ti}+{ }^{208} \mathrm{~Pb}$ are also shown.

capture cross section $\sigma_{c}$ (Fig. 2) and (ii) the prominence of the fast mass-asymmetric components in Fig. 3. Suppression of $\sigma_{\text {fis }}$ clearly results from both DIC and fast QF, both with short timescales, small rotation angles, and limited mass drift towards symmetry.

Yield at mass symmetry can result not only from fusion fission (which gives information on $P_{\mathrm{CN}}$ ) but also from slow $\mathrm{QF}$ - having timescales longer than fast QF. Thus $P_{\mathrm{CN}}=\sigma_{\mathrm{FF}} / \sigma_{c}<\left(\sigma_{\mathrm{FF}}+\sigma_{\mathrm{SQF}}\right) / \sigma_{c}$, where $\sigma_{\mathrm{FF}}, \sigma_{\mathrm{SQF}}$ are the cross sections for fusion fission and slow QF. Therefore to extract quantitative values of $P_{\mathrm{CN}}$, the contributions of quasifission to the mass-symmetric region should be subtracted. Previous work for ${ }^{50} \mathrm{Ti}+{ }^{208} \mathrm{~Pb}$ [20] has used the angular distribution of mass-symmetric fission to extract the $P_{\mathrm{CN}}$ values shown in Fig. 4, but since assumptions had to be made about quasifission angular distributions, it was "difficult to make meaningful estimates of the uncertainties in $P_{\mathrm{CN}}$ " [20]. In this Letter, the contribution from quasifission was reduced by fitting the experimental $M_{R}$ spectra (Fig. 1) with two functions, comprising an asymmetric $U$-shaped background function (shown by thin blue lines) representing quasifission [11], and a Gaussian representing the peak near mass-symmetry, representing fusion fission and the slowest quasifission events. The shape of the background function was based on measurements for the reaction ${ }^{54} \mathrm{Cr}+{ }^{204} \mathrm{~Pb}$ having fewer magic numbers than those studied here, and showing no masssymmetric peak [11] [see Fig. 1(p) and Supplemental Material [31]].

The ratio of the cross section of the Gaussian component $\sigma_{\text {sym }}$ to the capture cross section $\sigma_{c}$ is designated $P_{\text {sym }}$ [31]. It represents an upper limit to $P_{\mathrm{CN}}$ because at the higher energies, the fitted Gaussian was centered at $M_{R}>0.5$, indicating that a slow quasifission component is still 
included in $\sigma_{\mathrm{sym}} . P_{\mathrm{sym}}$ is shown as a function of excitation energy $E_{x}$ in Fig. 4.

Two important characteristics are clearly seen in the present data, which reflect the qualitative conclusions reached by direct inspection of the experimental MADs: (i) the fraction of slow symmetric-peaked fission reduces with $Z_{P}$, by a factor of 2.5 from ${ }^{48} \mathrm{Ca}$ to ${ }^{50} \mathrm{Ti}$, and by a further factor of 6 to ${ }^{54} \mathrm{Cr}$; (ii) $P_{\text {sym }}$ has its maximum value at the lowest energy, corresponding to near- and subbarrier collisions. It is likely that with increasing $E_{x}, P_{\mathrm{CN}}$ itself will fall more rapidly than $P_{\text {sym }}$, since the quasifission component near symmetry increases with beam energy as demonstrated by the mass-angle correlations (Fig. 1) and the observation of an increasing offset of the fitted Gaussian from $M_{R}=0.5$.

Calculated values of $P_{\mathrm{CN}}$ are shown in Fig. 4, from the semiempirical angular momentum dependent expression proposed by Zagrebaev and Greiner specifically for ${ }^{208} \mathrm{~Pb}$ reactions [19]. The fall in $P_{\mathrm{CN}}$ with $Z_{P}$ matches the experimental $P_{\text {sym }}$ reasonably well; however the decrease in $P_{\mathrm{CN}}$ as $E_{x}$ falls is opposite to the present experimental trend. The calculations are based on a diffusion model, which considers thermal shape fluctuations (diffusion) as the mechanism to transport the composite system over the potential surface to the compact compound nucleus shape. Diffusion implies that $P_{C N} \rightarrow 0$ as $E_{x} \rightarrow 0$. The experimental trend suggests that cold fusion is not driven by a thermal diffusion process. Instead a microscopic approach to obtain a nuclear structure dependent energy dissipation may give a better framework to explain the new experimental data [9-12] on fusion dynamics in reactions with ${ }^{208} \mathrm{~Pb}$ nuclei.

In summary, extensive measurements of mass-angle distributions and cross sections for ${ }^{48} \mathrm{Ca},{ }^{50} \mathrm{Ti}$, and ${ }^{54} \mathrm{Cr}+$ ${ }^{208} \mathrm{~Pb}$ have been presented. They show a systematic decrease in the fraction of mass-symmetric fission as a function of increasing projectile charge and beam energy. This is correlated with an increase in nonequilibrium deepinelastic and fast quasifission processes. After subtracting a quasifission contribution from the mass-symmetric region based on the measured data for a similar system involving fewer magic numbers, upper limits have been extracted for the probability $P_{\mathrm{CN}}$ of forming a compact compound nucleus. They show $\mathrm{P}_{\mathrm{CN}}$ for ${ }^{50} \mathrm{Ti}$ is at least 2.5 times smaller than for ${ }^{48} \mathrm{Ca}$, whilst ${ }^{54} \mathrm{Cr}$ is over 15 times smaller. For all reactions, $P_{\mathrm{CN}}$ is highest at the lowest beam energy, opposite to the trends of a diffusion-based model. This dependence, and the known sensitivity to closed shells in the colliding nuclei [9-12] suggests that diffusion is not the main mechanism that drives SHE formation in fusion reactions with ${ }^{208} \mathrm{~Pb}$ nuclei, and a microscopic approach to energy dissipation and fluctuations is required.

The authors acknowledge the Australian Research Council for support through Discovery Grants No. DP140101337,
No. DP160101254, No. DP170102318, No. FL110100098, and No. DE140100784. Financial support from the NCRIS HIA capability for operation of the Heavy Ion Accelerator Facility is acknowledged. The authors acknowledge the support of the German Academic Exchange Service (DAAD) via funds of the German Federal Ministry of Education and Research (BMBF).

*kaushik@vecc.gov.in

On leave from Variable Energy Cyclotron Centre, 1/AF, Bidhan Nagar, Kolkata 700064, India

david.hinde@anu.edu.au

*Present address: CSIRO Sorting and Sensing, Mineral Resources, Building 67, ANSTO, Lucas Heights, Australia. ${ }^{\S}$ Present address: Department of Physics, School of Mathematical and Physical Sciences, Central University of Kerala, Kasaragod 671314, India.

"Present address: Advanced Technology Institute, University of Surrey, Guildford, Surrey GU2 7XH, United Kingdom.

[1] A. Sobiczewski, F. A. Gareev, and B. N. Kalinkin, Phys. Lett. 22, 500 (1966).

[2] W. D. Myers and W. J. Swiatecki, Nucl. Phys. 81, 1 (1966).

[3] U. Mosel and W. Greiner, Z. Phys. 222, 261 (1969).

[4] M. Bender, K. Rutz, P. G. Reinhard, J. A. Maruhn, and W. Greiner, Phys. Rev. C 60, 034304 (1999).

[5] Y. Oganessian, J. Phys. G 34, R165 (2007).

[6] W. U. Schröder, J. R. Huizenga, Damped nuclear collisions, in Treatise on Heavy Ion Science, edited by D. A. Bromley (Plenum Press, New York, 1984), Vol. 2, Chap. 3.

[7] E. Williams, K. Sekizawa, D. J. Hinde, C. Simenel, M. Dasgupta, I. P. Carter, K. J. Cook, D. Y. Jeung, S. D. McNeil, C. S. Palshetkar, D. C. Rafferty, K. Ramachandran, and A. Wakhle, Phys. Rev. Lett. 120, 022501 (2018).

[8] J. Tõke, B. Bock, G. X. Dai, A. Gobbi, S. Gralla, K. D. Hildenbrand, J. Kuzminski, W. F. J. Müller, A. Olmi, and H. Stelzeret, Nucl. Phys. A440, 327 (1985).

[9] C. Simenel, D. J. Hinde, R. du Rietz, M. Dasgupta, M. Evers, C. J. Lin, D. H. Luong, and A. Wakhle, Phys. Lett. B 710, 607 (2012).

[10] J. Khuyagbaatar, D. J. Hinde, I. P. Carter, M. Dasgupta, Ch. E. Düllmann, M. Evers, D. H. Luong, R. du Rietz, A. Wakhle, E. Williams, and A. Yakushev, Phys. Rev. C 91, 054608 (2015).

[11] G. Mohanto, D. J. Hinde, K. Banerjee, M. Dasgupta, D. Y. Jeung, C. Simenel, E. C. Simpson, A. Wakhle, E. Williams, I. P. Carter, K. J. Cook, D. H. Luong, C. S. Palshetkar, and D. C. Rafferty, Phys. Rev. C 97, 054603 (2018).

[12] J. Khuyagbaatar et al., Phys. Rev. C 97, 064618 (2018).

[13] Y. T. Oganessian et al., Phys. Rev. Lett. 109, 162501 (2012).

[14] J. Roberto, C. Alexander, R. Boll, J. Burns, J. Ezold, L. Felker, S. Hogle, and K. Rykaczewski, Nucl. Phys. A944, 99 (2015).

[15] J. Khuyagbaatar, EPJ Web Conf. 163, 00030 (2017).

[16] S. Hofmann et al., GSI Scientific Report-2011, 205, 2012.

[17] Yu. Ts. Oganessian et al., Phys. Rev. C 79, 024603 (2009).

[18] S. Hofmann, D. Ackermann, S. Antalic, V. F. Comas, S. Heinz, J. A. Heredia, F. P. Heßberger, J. Khuyagbaatar, B. Kindler, I. Kojouharov, M. Leino, B. Lommel, R. Mann, 
K. Nishio, A. G. Popeko, S. Saro, J. Uusitalo, M. Venhart, and A. V. Yeremin, GSI Scientific Report-2008, 131, 2009.

[19] V. Zagrebaev and W. Greiner, Phys. Rev. C 78, 034610 (2008).

[20] R. S. Naik, W. Loveland, P. H. Sprunger, A. M. Vinodkumar, D. Peterson, C. L. Jiang, S. Zhu, X. Tang, E. F. Moore, and P. Chowdhury, Phys. Rev. C 76, 054604 (2007).

[21] W. J. Światecki, K. Siwek-Wilczyńska, and J. Wilczynski, Phys. Rev. C 71, 014602 (2005).

[22] K. Hagino, Phys. Rev. C 98, 014607 (2018).

[23] W. Loveland, J. Phys. Conf. Ser. 420, 012004 (2013).

[24] R. Yanez, W. Loveland, J. S. Barrett, L. Yao, B. B. Back, S. Zhu, and T. L. Khoo, Phys. Rev. C 88, 014606 (2013).

[25] A. C. Berriman, D. J. Hinde, M. Dasgupta, C. R. Morton, R. D. Butt, and J. O. Newton, Nature (London) 413, 144 (2001).

[26] D. J. Hinde and M. Dasgupta, Phys. Lett. B 622, 23 (2005).

[27] K. Nishio, S. Mitsuoka, I. Nishinaka, H. Makii, Y. Wakabayashi, H. Ikezoe, K. Hirose, T. Ohtsuki, Y. Aritomo, and S. Hofmann, Phys. Rev. C 86, 034608 (2012).

[28] J. Khuyagbaatar, K. Nishio, S. Hofmann, D. Ackermann, M. Block, S. Heinz, F. P. Hessberger, K. Hirose, H. Ikezoe, B. Kinlder, B. Lommel, H. Makii, S. Mitsuoka, I. Nishinaka, T. Ohtsuki, Y. Wakabayashi, and S. Yan, Phys. Rev. C 86, 064602 (2012).

[29] R. G. Thomas, D. J. Hinde, D. Duniec, F. Zenke, M. Das gupta, M. L. Brown, M. Evers, L. R. Gasques, M. D. Rodriguez, and A. Diaz-Torres, Phys. Rev. C 77, 034610 (2008).

[30] R. du Rietz, E. Williams, D. J. Hinde, M. Dasgupta, M. Evers, C. J. Lin, D. H. Luong, C. Simenel, and A. Wakhle, Phys. Rev. C 88, 054618 (2013).

[31] See Supplemental Material at http://link.aps.org/ supplemental/10.1103/PhysRevLett.122.232503 for an extended discussion of the experimental setup and analysis procedure, which also includes Refs. [32,33].

[32] I. M. Itkis, E. M. Kozulin, M. G. Itkis, G. N. Knyazheva, A. A. Bogachev, E. V. Chernysheva, L. Krupa, Yu. Ts. Oganessian, V. I. Zagrebaev, A. Ya. Rusanov, F. Goennenwein, O. Dorvaux, L. Stuttgé, F. Hanappe, E. Vardaci, and E. de Goés Brennand, Phys. Rev. C 83, 064613 (2011).

[33] E. M. Kozulin, G. N. Knyazheva, I. M. Itkis, M. G. Itkis, A. A. Bogachev, E. V. Chernysheva, L. Krupa, F. Hanappe, O. Dorvaux, L. Stuttgé, W. H. Trzaska, C. Schmitt, and G. Chubarian, Phys. Rev. C 90, 054608 (2014).

[34] D. J. Hinde, R. du Rietz, M. Dasgupta, R. G. Thomas, and L. R. Gasques, Phys. Rev. Lett. 101, 092701 (2008).
[35] D. J. Hinde, D. Hilscher, H. Rossner, B. Gebauer, M. Lehmann, and M. Wilpert, Phys. Rev. C 45, 1229 (1992).

[36] J. Cabrera, Th. Keutgen, Y. El Masri, Ch. Dufauquez, V. Roberfroid, I. Tilquin, J. Van Mol, R. Régimbart, R. J. Charity, J. B. Natowitz, K. Hagel, R. Wada, and D. J. Hinde, Phys. Rev. C 68, 034613 (2003).

[37] D. J. Hinde, D. Y. Jeung, E. Prasad, A. Wakhle, M. Dasgupta, M. Evers, D. H. Luong, R. du Rietz, C. Simenel, E. C. Simpson, and E. Williams, Phys. Rev. C 97, 024616 (2018).

[38] E. V. Prokhorova, A. A. Bogachev, M. G. Itkis, I. M. Itkis, G. N. Knyazheva, N. A. Kondratiev, E. M. Kozulin, L. Krupa, Yu. Ts. Oganessian, I. V. Pokrovsky, V. V. Pashkevich, and A. Ya. Rusanov, Nucl. Phys. A802, 45 (2008).

[39] B. B. Back, R. R. Betts, J. E. Gindler, B. D. Wilkins, S. Saini, M. B. Tsang, C. K. Gelbke, W. G. Lynch, M. A. McMahan, and P. A. Baisden, Phys. Rev. C 32, 195 (1985).

[40] R. Bock, Y. T. Chu, M. Dakowski, A. Gobbi, E. Grosse, A. Olmi, H. Sann, D. Schwalm, U. Lynen, W. Müller, S. Bjørnholm, H. Esbensen, W. Wülfli, and E. Morenzoni, Nucl. Phys. A388, 334 (1982).

[41] A. J. Pacheco, J. O. Fernandez Niello, D. E. DiGregorio, M. di Tada, J. E. Testoni, Y. Chan, E. Chavez, S. Gazes, E. Plagnol, and R. G. Stokstad, Phys. Rev. C 45, 2861 (1992).

[42] H.-G. Clerc, J. G. Keller, C.-C.Sahm, K.-H.Schmidt, H. Schulte, and D. Vermeulen, Nucl. Phys. A419, 571 (1984).

[43] K. Hagino, N. Rowley, and A. T. Kruppa, Comput. Phys. Commun. 123, 143 (1999).

[44] J. O. Newton, R. D. Butt, M. Dasgupta, D. J. Hinde, I. I. Gontchar, C. R. Morton, and K. Hagino, Phys. Rev. C 70, 024605 (2004).

[45] M. G. Itkis, A. A. Bogachev, I. M. Itkis, J. Kliman, G. N. Knyazheva, N. A. Kondratiev, E. M. Kozulin, L. Krupa, Yu. Ts. Oganessian, I. V. Pokrovsky, E. V. Prokhorova, and A. Ya. Rusanov, Nucl. Phys. A787, 150 (2007).

[46] E. M. Kozulin, G. N. Knyazheva, K. V. Novikov, I. M. Itkis, M. G. Itkis, S. N. Dmitriev, Yu. Ts. Oganessian, A. A. Bogachev, N. I. Kozulina, I. Harca, W. H. Trzaska, and T. K. Ghosh, Phys. Rev. C 94, 054613 (2016).

[47] V. E. Viola, K. Kwiatkowski, and M. Walker, Phys. Rev. C 31, 1550 (1985).

[48] D. J. Hinde, J. R. Leigh, J. J. M. Bokhorst, J. O. Newton, R. L. Walsh, and J. W. Boldeman, Nucl. Phys. A472, 318 (1987). 